\title{
SEMICONDUCTOR DETECTORS FOR OBSERVATION OF MULTI-MEV PROTONS AND IONS PRODUCED BY LASERS
}

\author{
Krása J ${ }^{a}{ }^{*}$, Klír D. ${ }^{b}$, De Marco M. ${ }^{a, e}$, Cikhardt J. ${ }^{b}$, Velyhan ${ }^{a}$, \\ ŘezÁč K. ${ }^{b, c}$, Pfeifer M. ${ }^{a}$, Krouský E. ${ }^{a}$, RyĆ L. ${ }^{d}$, Dostál J. ${ }^{c}$, \\ Kaufman J. ${ }^{a}$, Ullschmied J. ${ }^{c}, \quad$ Limpouch J. ${ }^{e}$ \\ ${ }^{a}$ Institute of Physics of the CAS, Na Slovance 2, 12121 Prague, Czech Republic \\ ${ }^{b}$ Faculty of Electrical Engineering, CTU in Prague, Technická 2, 166 27 Prague, Czech Republic \\ ${ }^{c}$ Institute of Plasma Physics of the CAS, Za Slovankou 3, 18200 Prague, Czech Republic \\ $d$ Institute of Plasma Physics and Laser Microfusion, EURATOM Association, Warsaw, Poland \\ e Faculty of Nuclear Sciences and Physical Engineering, CTU in Prague, Břehová 2, 11519 Prague, \\ Czech Republic \\ * krasa@fzu.cz
}

\begin{abstract}
The application of time-of-flight Faraday cups and $\mathrm{SiC}$ detectors for the measurement of currents of fast ions emitted by laser-produced plasmas is reported. Presented analysis of signals of ion detectors reflects the design and construction of the detector used. A similarity relation between output signals of ion collectors and semiconductor detectors is established. Optimization of the diagnostic system is discussed with respect to the emission time of electromagnetic pulses interfering with signals induced by the fastest ions accelerated up to velocities of $10^{7} \mathrm{~m} / \mathrm{s}$. The experimental campaign on laser-driven ion acceleration was performed at the PALS facility in Prague.
\end{abstract}

Keywords: laser-accelerated ions, ion collectors, SiC detectors, similarity relations, electromagnetic pulse. .

\section{Introduction}

Laser-produced plasmas are employed as secondary sources of X-ray radiation, beams of electrons as well as ions capable for various applications. Laseracceleration of ions represents an effective technique that allows accelerating of all ionized elements for various applications including acceleration of ions entering to fusion reactions with chosen elements in a pitcher-catcher target scheme [1]. A stream of fast ions expanding into an interaction chamber to impact on a secondary target should have optimal energy distribution with respect, for example, to the crosssection of required fusion reactions. The ion expansion is detected with the use of a variety of detectors whose signals are proportional to the number of impacted particles, or their velocity, eventually deposited energy into the sensitive detector's bulk [2]. Due to the plasma rarefaction caused by its expansion, the ions enter to a phase, where no collisions between ions occur and their charge-states are frozen. Then the time-of-flight spectra of ions exhibit a well-defined dependence on the detector's distance from a target, which allows us rescaling the ion current observed at a distance $L_{2}$ from a target to another distance $L_{1}$. The procedure needed for the ion current rescaling from one to another distance is based on similarity relations [2]:

$$
t_{L 1}=(L 1 / L 2) \times t_{L 2}
$$

and

$$
j_{L 1}=(L 2 / L 1)^{3} \times j_{L 2} .
$$

The last relationship for currents allows us to rescale the signals of ion detectors to the same distance and collecting area and verify whether the time-resolved signals of ion collectors are identical. If the signals match themselves then recombination phenomena can be neglected at the chosen flight distances of observation and the analysis of ion currents based on a shifted Maxwell-Boltzmann velocity distribution can be accomplished 3, 4. Figure 1 shows a schematic diagram of the experiment arrangement for cross-calibration of various ion detectors by a stream of ions emitted by a laser-produced plasma. A ring detector has an inner hole allowing the passage of ions to the second detector. This configuration overwhelms an undesirable effect of the anisotropy in ion emission [5] and makes an application of the similarity relationship 2 possible.

This contribution is devoted to the application of silicon carbide semiconductors as detectors of multi$\mathrm{MeV}$ protons and deuterons which have sufficient kinetic energy to enter fusion reactions as, for example, ${ }^{2} \mathrm{D}(\mathrm{d} ; \mathrm{n}){ }^{3} \mathrm{He},{ }^{7} \mathrm{Li}(\mathrm{d} ; \mathrm{n}){ }^{8} \mathrm{Be}$, and ${ }^{11} \mathrm{~B}(\mathrm{p} ; \alpha) 2 \alpha$, which are driven by lasers as well as z-pinches [1, 6, 7].

\section{Generation and detection of ions}

Deuteron-producing experiments were carried out on the PALS at the Institute of Plasma Physics in Prague. 


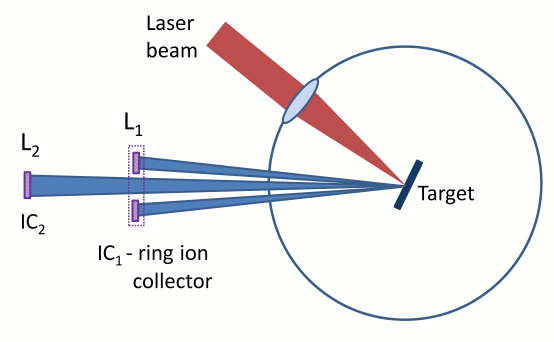

Figure 1. Schematic diagram of the experiment and an example of ion current densities $j$ measured with ion detectors $I C_{1}$ (ring ion collector) and $I C_{2}$ located at distances $L_{1}$ and $L_{2}$ from the target, respectively

At the fundamental wavelength of $1.315 \mu \mathrm{m}$, the PALS laser was capable of delivering the pulse with a duration of 300 ps (FWHM) and an energy of about $500 \mathrm{~J}$ on a target. The corresponding intensity producing the plasma on deuterated-polyethylene targets was $I_{\mathrm{L}} \approx 3 \times 10^{16} \mathrm{~W} / \mathrm{cm}^{2}$. The thickness of targets varied from 200 to $500 \mu \mathrm{m}$.

The emission of ions was observed with the use of ion collectors (ICs), silicon-carbide ( $\mathrm{SiC}$ ) time-offlight detector positioned in the far expansion zone, i.e., outside the recombination zone. The ion collector positioned $1.51 \mathrm{~m}$ far from the target was screened with an $\mathrm{Al}$ foil of $6 \mu \mathrm{m}$ in thickness to suppress a photopeak in the IC signal induced by XUV radiation. The suppression of the photopeak of a $150 \mathrm{~ns}$ duration allowed us to detect $\mathrm{MeV}$ protons reaching the IC $50 \mathrm{~ns}$ after the laser pulse 8. On the other hand, the $6-\mu \mathrm{m} \mathrm{Al}$ foil absorbed slower ions and, thus, limited the range of energy spectra to energies higher than $500 \mathrm{keV}$ and $600 \mathrm{keV}$ for protons and deuterons, respectively.

The SiC detectors are blind to visible and infrared radiation. This characteristics is advantageous because the laser-produced plasma emits very intense radiation with wavelength $\lambda>380 \mathrm{~nm}$. Distance of the $\mathrm{SiC}$ detector to the target was $1.54 \mathrm{~m}$. The internal structure of the $\mathrm{SiC}$ detector and the geometry of its front electrode is shown elsewhere [9, 10]. The $\mathrm{SiC}$ detector is composed of 4 layers: $\mathrm{Ni}_{2} \mathrm{Si}$ contact (200-nm thick front electrode), 100- $\mu$ m epitaxial layer, 150-nm n+buffer layer localized above the active $\mathrm{n}++4 \mathrm{H}-\mathrm{SiC}$ layer of $430 \mu \mathrm{m}$ in thickness, and the last layer is Ni-ohmic-contact. The used SiC detector was biased at $300 \mathrm{~V}$.

\section{Results and discussion}

The signal $S_{x}$ of a time-of-flight detector measuring the impacted ions in $x$-direction depends on its response to their number, velocity or energy, which

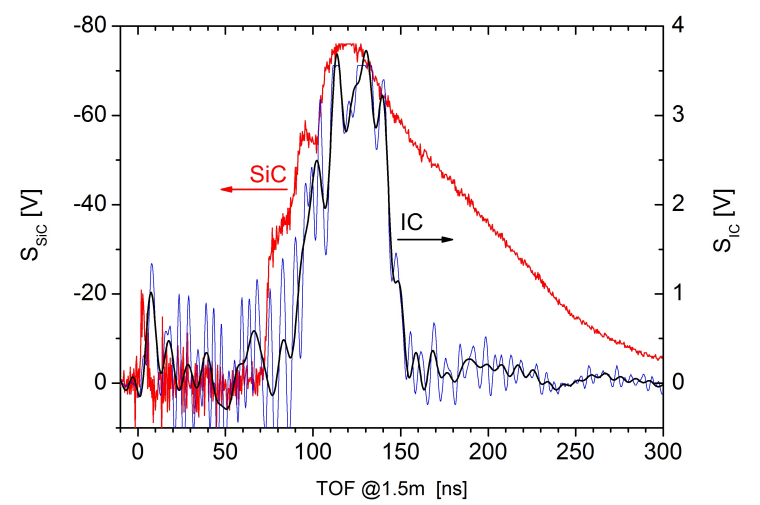

Figure 2. Signals of $\mathrm{SiC}$ and $\mathrm{IC}$ detectors related to the distance of $1.5 \mathrm{~m}$ to the target. Ions were emitted by a $C D_{2}$ target exposed to the laser intensity of $3 \times 10^{16}$ $W / \mathrm{cm}^{2}$.

can be expressed as follows: $S_{x}(L, t, \alpha) \propto v^{\alpha} f(\mathbf{v}) d \mathbf{v}$, where $v$ is the velocity of ions in the direction of observation, $f(\mathbf{v})$ is the 3 -dimensional velocity distribution function and $\alpha=1$ if the response is proportional to the stream (current) of incident particles - applicable to ion collectors, and $\alpha=2$ if the response is proportional to the deposited energy by particles - applicable to semiconductor detectors [2]. Both the types of ion detectors give information on charge density carried by ions if their signals are rescaled to a unit of sensitive area of detectors and to the same distance from the irradiated target. Simplifying the equations used to model signals of detectors, we obtain simple relationships between the charge density carried by ions, $n_{Q}$, and output signal of ion collectors, $S_{\text {IC }}$, as well as $\mathrm{SiC}$ detectors, $S_{\mathrm{SiC}}$ :

$$
n_{Q}(L, t) \approx S_{\mathrm{IC}}(L, t) t / L \approx S_{\mathrm{SiC}}(t) t^{2} / L^{2},
$$

where $L / t=v(t)$ is the velocity of ions passing the distance $L$ at a time $t$. Relationship 3 establishes a similarity relation between signals of ion collectors and semiconductor detectors.

Figure 2 shows a comparison of signals $S_{\text {IC }}(t)$ and $S_{\mathrm{SiC}}(t)$, where $\mathrm{SiC}$ detector is placed in the $\mathrm{IC}_{2}$ place. The reference distance from the target is $1.5 \mathrm{~m}$. It is evident that the shape of the signal $S_{\text {IC }}(t)$ differs from the signal $S_{\mathrm{SiC}}(t)$ because $S_{\mathrm{IC}}(t)$ is proportional to the velocity and charge of detected ions while the signal $S_{\mathrm{SiC}}(t)$ is proportional to the energy of ions. The IC detected only protons, deuterons and carbon ions being able to pass through the $\mathrm{Al}$ foil placed in front of the IC. Thus, the IC signal turns down at about 150 ns for ionized species emitted by the $\mathrm{CD}_{2}$ plasma. Corresponding kinetic energies of ions stopped in the Al foil were calculated using the Stopping and Range of Ions in Matter (SRIM) code[11.

The TOF signals allow us to determine the fastest velocity of detected ions if the electromagnetic pulse 


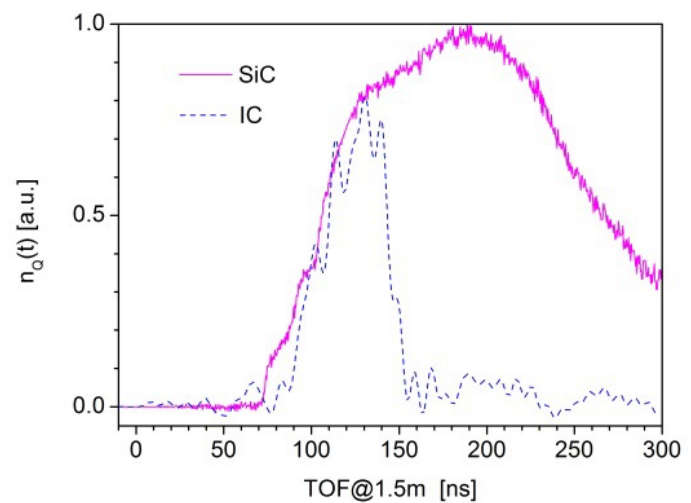

Figure 3. Comparison of output signals of SiC detector and ion collector, which were rescaled to ion charge density using relationship 3 , see Figure 2

(EMP) does not strongly interfere with the detector's signal, as the row signal (blue line) $S_{\mathrm{IC}}(t)$ shows in Figure 2; the black line is the smoothed $S_{\mathrm{IC}}(t)$ signal. The EMP is produced in the interaction chamber during generation and expansion of the laser-produced plasma [12. Thus, in this laser-plasma shot, the protons, which are the fastest of all ions emitted by the laser-produced plasma, reached energy of $2.5 \mathrm{MeV}$, as determined from $S_{\mathrm{SiC}}(t)$.

The rescaling $S_{\mathrm{SiC}}(t)$ and $S_{\mathrm{IC}}(t)$ to the charge density of ions with the use of (1) is shown in Figure 3 Since the rescaled signals match themselves, Figure 3 confirms that the $\mathrm{SiC}$ detector can be successfully applied as an ion detector in plasma experiments. We note that the rescaling of $S_{\mathrm{IC}}(t)$ and $S_{\mathrm{SiC}}(t)$ to $n_{\mathrm{Q}}(t)$ reduces the noise which occurs on detector signals due to the interference with an electromagnetic pulse (EMP) during the first period of a few hundreds of nanoseconds of the observation of ions.

\section{Conclusions}

Although the $\mathrm{SiC}$ detector used in the experiment are not fully valuable for the measurement of the lowenergy protons and ions as the IC, as they cannot penetrate through the input 200-nm $\mathrm{Ni}_{2} \mathrm{Si}$ layer, this $\mathrm{SiC}$ detector is a valuable tool for detection of multi$\mathrm{MeV}$ protons. The advantage of a $\mathrm{SiC}$ detector is that it is more sensitive for fast ions than the ion collector [8] 10].

\section{Acknowledgements}

The research leading to these results has received funding from the Czech Science Foundation (Grant No.16-07036S), European Regional Developmentthe project ELI: Extreme Light Infrastructure (CZ.02.1.01/0.0/0.0/15_008/0000162), and the Czech Republic's Ministry of Education, Youth and Sports (LD14089).

\section{References}

[1] D. Klir, et al. Efficient neutron production from sub-nanosecond laser pulse accelerating deuterons on target front side, Phys. Plasmas, 229(9): 093117, 2015.

[2] J. Krása. Gaussian energy distribution of fast ions emitted by laser-produced plasmas. Applied Surface Science 272: 46-49, 2013.

[3] J. Lorusso, et al. Charge losses in expanding plasma created by an XeCl laser. Appl. Phys. Lett. 86(8): 081501, 2005.

[4] M. Comet, et al. Absolute energy distributions of $\mathrm{Al}$, $\mathrm{Cu}$, and $\mathrm{Ta}$ ions produced by nanosecond lasergenerated plasmas at $1013 \mathrm{~W} \mathrm{~cm}^{-2}$. J. Appl. Phys. 119(1): 013301, 2016.

[5] J. Krása, et al. The effect of laser-produced plasma expansion on the ion population. Appl. Phys. Lett. 75(17): 2539-2541, 1999.

[6] D. Picciotto, et al. Boron-Proton nuclear-fusion enhancement induced in boron-doped silicon targets by low-contrast pulsed laser. Phys. Rev. X 4(3): 031030, 2014.

[7] D. Klir, et al. Deuterium z-pinch as a powerful source of multi-MeV ions and neutrons for advanced applications. Phys. Plasmas, 230(2): 032702, 2016.

[8] D. Margarone, et al. Full characterization of laser-accelerated ion beams using Faraday cup, silicon carbide, and single-crystal diamond detectors. J. Appl. Phys. 109(10): 103302, 2011.

[9] L. Ryć, et al.: Application of a single-crystal CVD diamond detector for simultaneous measurement of ions and X-rays from laser plasmas. Radiat. Eff. Defects Solids 165(6-10): 481-487, 2010.

[10] L. Ryć, et al. Measurement of ion emission from plasmas obtained with a $600 \mathrm{fs} \mathrm{KrF}$ laser. Phys. Scr. T161: 014032, 2014.

[11] J. F. Ziegler, et al. SRIM - The Stopping and Range of Ions in Matter. (available at: www.srim.org)

[12] M. De Marco, et al. Electromagnetic pulses produced by expanding laser-produced Au plasma. Nucleonika 60(2): 239-243, 2015. 